\title{
Programa Bolsa Família e educação escolar: uma abordagem de dados em painel
}

\author{
Patrícia Verônica Pinheiro Sales Lima \\ Universidade Federal do Ceará - Fortaleza - Ceará - Brasil \\ Joyciane Coelho Vasconcelos \\ Universidade Federal do Ceará - Fortaleza - Ceará - Brasil
}

\section{Resumo}

Este artigo tem como objetivo avaliar se, e de que forma, o Programa Bolsa Família (PBF) contribuiu para melhorar os indicadores de educação infantil e ensino fundamental nos Estados Brasileiros. Com a estimação de modelos de regressão com dados em Painel para período de 2005 a 2014 espera-se verificar se os recursos do PBF foram capazes de influenciar o comportamento dos indicadores educacionais nas unidades brasileiras. Logo, pretende-se potencializar o impacto do PBF na promoção de uma educação mais inclusiva e de melhor qualidade. Despertar os Estados para a necessidade de criar mecanismos que favoreçam a efetividade das políticas sociais realizadas pelo Governo Federal. Os resultados deste artigo revelam que a situação da educação esta melhorando no Brasil como um todo. Mas, em alguns Estados isso ocorre de forma lenta e verifica-se são necessários vários anos para se alcançar uma situação menos desigual na educação entre as Unidades Federativas.

Palavras-Chave: Políticas públicas. Educação. Programa Bolsa Família.

\section{Bolsa Família Program and Schooling: A Panel Data Approach}

\begin{abstract}
This article aims at evaluating, and in what way, the Bolsa Família Program (PBF) contributed to improving the indicators of early childhood education and basic education in the Brazilian States. With the estimation of regression models with data in Panel for period from 2005 to 2014 it is expected to verify if the resources of the PBF were able to influence the behavior of the educational indicators in Brazilian units. Therefore, it is intended enhance the impact of the PBF in promoting a more inclusive and better quality education. To awaken states to the need to create mechanisms that favor the effectiveness of social policies carried out by the Federal Government. The results of this article reveal that the education situation is improving in Brazil as a whole. But in some states this occurs slowly and it turns out that it takes several years to reach a less unequal situation in education between the Federative Units.
\end{abstract}

Keywords: Public policies. Education. Bolsa Família Program. 


\section{Programa Bolsa Familia y Educación Escolar: Un Enfoque de Datos en Panel}

\section{Resumen}

Este artículo tiene como meta evaluar si, y de qué camino, el Programa Bolsa Familia (PBF) contribuyó a mejorar los indicadores de educación infantil y enseñanza primaria en los Estados Brasileños. Con la estimación de modelos de regresión con datos en Panel para período de 2005 a 2014 si desea comprobar si los recursos del PBF fueron capaces de influencia el comportamiento de los indicadores educativos en las unidades brasileñas. Por lo tanto, si desea potenciar el impacto del PBF en la promoción de una educación más inclusiva y de mejor calidad. Despertar a los Estados para la necesidad de crear mecanismos que favorezcan la efectividad de las políticas sociales llevado a cabo por el Gobierno Federal. Los resultados de este artículo revelan que la situación de la educación es mejorando en Brasil como un todos. Pero en algunos Estados esto ocurre de camino lento y si desea son necesarios varios años para alcanzar una situación mas igualdad en la educación en las unidades brasileñas.

Palabras-Clave: Políticas públicas. Educación. Programa Bolsa Familia.

\section{Introdução}

Nos últimos anos o governo tem se preocupado em adotar uma postura voltada para o desenvolvimento sustentável. Nesse sentido, as políticas públicas vêm sendo elaboradas com base nos pilares social, econômico e ambiental, embora ainda seja percebida a ênfase nas dimensões social e econômica. O Programa Bolsa Família (PBF) é um exemplo dessa postura, haja vista seus objetivos: impactar na desigualdade de renda, na pobreza, na educação, na diminuição no trabalho infantil e no aumento da segurança alimentar.

O PBF abrange 16 milhões de alunos acompanhados a cada dois meses que abrangem, em média, 50 milhões de famílias, o que representa cerca de $R \$ 25$ bilhões destinados ao programa, conforme informações do Ministério do Desenvolvimento Social e Agrário (MDSA,2014).

De acordo com os objetivos do PBF, destaca-se o aumento da frequência escolar das crianças. No entanto, ao se preocupar com a frequência das crianças às escolas tem-se, mesmo que de forma implícita, a preocupação com a formação de uma consciência que certamente contribuirá para a mudança de comportamento e, consequentemente, influenciará a relação homem - natureza. A evidente desigualdade no acesso à educação se reproduz nos índices que apontam para a qualidade do ensino. A nota média das escolas públicas no Sistema de Avaliação da Educação Básica (SAEB) é bem inferior a das privadas, "os alunos que terminam o Ensino Médio na escola pública demonstram, no seu conjunto, uma proficiência esperada para alunos da oitava série" (SOARES, 2004, p.155).

Sendo um dos objetivos do PBF promover o acesso de jovens e crianças à educação. $\mathrm{O}$ artigo tem como objetivo geral avaliar se, e de que forma, o Programa Bolsa Família contribuiu para melhorar os indicadores de educação infantil e ensino fundamental nos Estados Brasileiros. Esses indicadores baseiam-se nas seguintes variáveis: Total de crianças e adolescentes de 6 a 17 anos no ensino público da educação, quantidade de crianças e adolescentes de 6 a 17 anos acompanhados na frequência escolar, número de matrícula, nota do IDEB ( $5^{\circ}$ e $9^{\circ}$ ano) e taxa de aprovação. 
Ao buscar elementos que permitam avaliar de que forma o PBF cria oportunidades educacionais para a população, o artigo ganha importância e se diferencia dos demais por trazer possibilidades de análise a partir de uma visão interdisciplinar que requer a consulta a autores nas áreas de pedagogia, economia, sociologia, geografia, ciências políticas, administração pública, entre outras. Além disso, não são comuns avaliações do programa quanto a sua contribuição para a educação1. Por fim, pretende-se despertar os Estados para a necessidade de criar mecanismos que favoreçam a efetividade da política e assim, potencializar o impacto do PBF na promoção de uma educação mais inclusiva e de melhor qualidade.

Além desta introdução, o presente artigo está estruturado em mais cinco seções. Na próxima, são fornecidas evidências a respeito de como com o PBF vem contribuindo para mudar a educação. Na terceira seção, são descritos os dados e a metodologia utilizada na investigação. Os resultados das estimativas do impacto do programa Bolsa Família sobre o indicador educacional são apresentados e discutidos na quarta seção. E, na quinta e última seção, são expostas as conclusões do artigo.

\section{Programa Bolsa Família e Educação}

Nesta seção será abordada com o PBF vem contribuindo para mudar a educação. Sendo a educação um dos principais temas de discussões e reflexões. Sua importância deve-se ao fato de abranger temas sociais, econômicas, políticas e culturais dos diferentes países que veem na educação o pilar das mudanças estruturais da sociedade.

Segundo Pontili (2004), muitas pesquisas realizadas na área da economia têm apresentado a importância da escolaridade para a melhoria da qualidade de vida das pessoas. De acordo com a autora, o nível de escolaridade no Brasil é, em média, de 6,4 anos, considerado baixo em relação a outros países da América Latina. A partir dessa realidade, foram estabelecidas mudanças na forma de gestão de ensino público brasileiro, com o intuito de melhorar a qualidade do ensino e, principalmente, o interesse e permanência do aluno na escola.

De acordo Ney (2006), as desigualdades de oportunidades educacionais geram dois problemas básicos para a redução da desigualdade de renda no Brasil. 0 primeiro é que elas produzem grandes diferenças na qualidade da mão-de-obra que ingressa no mercado de trabalho, tendendo a gerar futuras disparidades de rendimentos. O segundo é que a pouca chance de os jovens mais pobres chegarem ao ensino médio limita a expansão da educação justo no nível em que sua taxa de retorno é maior. O efeito da escolaridade na renda depende do valor pago pelo mercado de trabalho a cada ano adicional de estudo, que é influenciado pela escassez relativa da oferta de mão-de-obra qualificada.

A percepção de que não se reduz desigualdade sem educação está implícita, mesmo de forma pouco perceptível, nos objetivos do PBF. Ao exigir frequência escolar das crianças cuja família é beneficiada o programa cria a expectativa de

\footnotetext{
${ }^{1}$ Melo e Duarte (2010) estudaram esse tema, porém apenas para o recorte da agricultura familiar no Nordeste. Outros autores analisaram mais intensamente os impactos do Bolsa-Família sobre o trabalho infantil (Ferro e Kassouf, 2005; Cacciamali et all., 2009) e os gastos de consumo (Resende, 2006). Ribeiro e Cacciamali (2012) investigam os impactos do programa Bolsa-Família sobre a frequência escolar para o ano de 2006.
} 
melhorar os indicadores de capital humano, mais especificamente aqueles referentes à dimensão educacional.

Desde implantação do PBF vários estudos foram realizados para estudar diversos aspectos de seu impacto. No entanto, o impacto do programa sobre a educação tem sido pouco explorado na literatura científica. Os trabalhos empíricos que abordam o Programa Bolsa Família se destinam, em sua maioria, à discussão do impacto desse programa sobre uma vasta gama de temáticas sociais, como o gasto familiar (consumo), o mercado de trabalho, a saúde, o trabalho infantil e a segurança alimentar.

Seguiram-se vários estudos que estimaram o impacto do PBF na redução da pobreza e da extrema pobreza, na desigualdade de renda, na permanência da criança na escola, entre outros. No tocante à desigualdade da renda, Soares et al (2010) mostram que $16 \%$ da redução observada entre 1999 e 2009 se deve ao Bolsa Família, muito embora a renda desse benefício não chegue a $0,8 \%$ da renda das famílias. A aparente contradição dessas participações é explicada pela focalização do programa, isto é, pelo fato de ser destinado aos mais pobres. Em relação à pobreza e à extrema pobreza, considerando as rendas per capita máximas para concessão do benefício básico e variável, esses pesquisadores, ao analisarem a evolução da queda observada desde 1990, apontam o aceleramento da queda com a presença do PBF.

Já em relação à frequência escolar, Silveira Neto (2010) aponta que o PBF é responsável pelo aumento de 2,2 pontos percentuais na frequência no meio urbano e de 3,0 pontos percentuais no meio rural. Em termos regionais, foi maior no Nordeste (2,2 pontos percentuais) do que na região Sudeste (1,5 pontos percentuais). Para se dimensionar a importância desses resultados, é preciso se levar em conta que a frequência escolar na região Sudoeste já era de 97,3\% e, no Nordeste, de 95,7\%.

Logo, a contribuição do PBF na redução da desigualdade deve estar atrelada à melhoria dos indicadores de educação. De acordo Ney (2006), as desigualdades de oportunidades educacionais geram dois problemas básicos para a redução da desigualdade de renda no Brasil. O primeiro é que elas produzem grandes diferenças na qualidade da mão-de-obra que ingressa no mercado de trabalho, tendendo a gerar futuras disparidades de rendimentos. O segundo é que a pouca chance de os jovens mais pobres chegarem ao ensino médio limita a expansão da educação justo no nível em que sua taxa de retorno é maior. $O$ efeito da escolaridade na renda depende do valor pago pelo mercado de trabalho a cada ano adicional de estudo, que é influenciado pela escassez relativa da oferta de mão-de-obra qualificada.

Seguindo tal linha, Glewwe e Kassouf (2010) estimaram o impacto do PBF sobre o total de matrículas do ensino fundamental e sobre as taxas de abandono e aprovação por meio de dados do Censo Escolar entre 1998 e 2005. Por meio do Censo Escolar destes anos não era possível identificar quais e quantos alunos eram beneficiados pelo programa em cada escola, de maneira que era possível saber somente se pelo menos um aluno da escola recebia o benefício. Utilizando regressões por escola, os autores mostram que o Programa Bolsa Família aumentou as matrículas de $1^{\mathrm{a}}$ a $4^{\mathrm{a}}$ série em $5,5 \%$ e de $5^{\mathrm{a}}$ a $8^{\mathrm{a}}$ série em $6,5 \%$, diminuiu as taxas de abandono escolar em 0,5 pontos percentual nas escolas de $1^{\mathrm{a}}$ a $4^{\mathrm{a}}$ série e em 0,4 por cento nas de $5^{\mathrm{a}}$ a $8^{\mathrm{a}}$ série e aumentou as taxas de aprovação em cerca de 0,9 ponto percentual de $1^{\mathrm{a}}$ a $4^{\mathrm{a}}$ série e 0,3 ponto percentual de $5^{\mathrm{a}}$ a $8^{\mathrm{a}}$ série. 
Neste mesmo contexto, Pellegrina (2011) avalia o impacto do PBF sobre o desempenho escolar e a matrícula dos alunos do estado de São Paulo. Utilizando as notas do SARESP de 2007 e 2009, além das notas de boletins escolares neste mesmo período, o autor estimou os possíveis impactos do programa sobre diferentes variáveis associadas à educação, todas ao nível de indivíduo. Por meio de diferentes métodos de matching e com o uso de diferenças-em-diferenças, o autor encontrou que o programa tem efeito sobre as variáveis que estão diretamente atreladas às condições impostas pelo PBF ao recebimento, mas nenhum efeito sobre as variáveis de desempenho escolar. $O$ autor encontrou evidências de que a participação no PBF reduz o abandono e aumenta a frequência escolar, entretanto parece não haver efeito sobre as notas em exames padronizados e sobre as notas do boletim escolar.

Oliveira e Soares (2013) mensuraram os efeitos do PBF na repetência. Concluíram que o aluno participante do programa possui uma probabilidade de repetência cerca de $11 \%$ menor do que os demais. Logo, o programa tem um impacto significativo sobre a progressão das crianças.

Uma análise preliminar dos estudos sobre o PBF permite fazer uma tipologia dos mesmos destacando três características: i) a variável impactada (renda, segurança alimentar, educação); ii) natureza dos dados (primários ou secundários) e iii) metodologia empregada (análises quantitativas, qualitativas, quantiqualitativas). Os estudos apresentam como ponto comum o fato de tratar o programa como uma intervenção federal dissociada das outras esferas do governo. Essa tendência tem criado lacunas de conhecimento quanto: i) à importância do ambiente institucional, especialmente no âmbito municipal, para a efetivação de políticas públicas e ii) capacidade de integração do PBF com outras intervenções governamentais no âmbito da educação.

Há diversas experiências internacionais de programas transferência de renda, os países da Europa foram os pioneiros na iniciação de programas de transferência de renda, adotados há mais de seis décadas pelo Reino Unido em 1948, seguido pela Finlândia em 1956 e Suécia em 1957.

Já na América Latina há vários países que utilizam programas sociais para tentar combater a pobreza, e não só no Brasil, destacando-se, por sua finalidade e relevância em análises comparativas internacionais, os programas existentes no México (Oportunidades) e no Chile (Chile Solidário). Nas Américas Central e do Sul, os programas de transferência condicionada de renda estão em vigor desde o final dos anos 1990. Os países México, Brasil, Honduras, Nicarágua e Colômbia possuem programas que oferecem subsídio financeiro para famílias pobres com crianças, condicionado a certos comportamentos educacionais e/ou de saúde.

Estimativas mostram que as taxas de matrícula cresceram aproximadamente $8 \%$ para meninas, e 4,5\% para meninos (SKOUFIAS, 2005). Efeito similar ocorre na redução da evasão escolar, com pesquisas encontrando reduções significativas para participantes do Bolsa Família, no Brasil, e Oportunidades, no México (Behrman, Parker e Todd, 2005; Janvry, Finan e Sadoulet, 2006).

Frota e Zimmermann (2010) fizeram uma comparação entre o Bolsa Família e o Oportunidades (mexicano) e levaram em consideração o contexto econômico e político bastante semelhante. Os autores afirmam que o formato institucional dos programas possuem condicionalidades voltadas a área da saúde e da educação, selecionam o público alvo definindo uma renda per capita, não concede o benefício a 
todos que dele necessitam, enfim, caracterizam-se pela focalização e rompem com princípios do direito humano. Concluíram que o Bolsa Família se apresenta mais coeso e mais integrado às políticas universais do sistema de proteção social. Há uma relação do Programa com os outros programas sociais do Fome Zero como, por exemplo, o Pronaf e a Economia popular solidária, o que, não acontece com o Programa mexicano já não tem promovido políticas que auxiliem na melhoria do quadro de vulnerabilidade social do país.

Berhrman et all. (2001) avaliam o impacto do Progresa sobre a frequência escolar, a repetência, a evasão e a taxa de retorno escolar entre as crianças que abandonaram a escola nos primeiros dois anos de operação desse programa (1998/1999). Os beneficiários do programa apresentaram maiores taxas de frequência às aulas, menor índice de repetência, menor nível de evasão e maior taxa de retorno entre as crianças que abandonaram a escola, em relação às crianças do grupo de controle. Um resultado ressaltado pelos autores se refere à notável redução de evasão escolar entre os beneficiários aptos a transitarem do ciclo escolar básico para o ciclo secundário. Os impactos do Progresa para meninos e meninas são similares na etapa do ensino primário, porém o programa apresenta melhores resultados no combate à evasão entre o ciclo básico e secundário para os meninos.

A partir dos estudos empíricos sobre os efeitos dos programas de transferência de renda condicional e não condicional, é possível concluir que existem muitos benefícios proporcionados por esses programas, principalmente para os mais pobres. Não significa que todos os programas sejam eficientes ou que exista algum livre de falhas. É preciso expandir as pesquisas sobre os resultados e construir modelos ainda mais eficientes, delimitando quais estratégias são eficazes e quais precisam ser reformuladas ou descartadas.

\section{Mensurando Educação}

A obtenção de dados sobre educação, especialmente se o interesse é selecionar indicadores comparáveis ao longo do tempo e entre Estados, requer, geralmente, o uso de fontes secundárias de dados. Pesquisadores que realizam análises a partir de fontes secundárias geralmente deparam-se com ausência de informações para algumas unidades de observação, alterações metodológicas na coleta de dados de um ano para o outro, inexistência de indicadores valiosos no contexto teórico do tema de estudo. Os dois entraves foram observados nessa pesquisa acrescentando-se, ainda, a dificuldade na operacionalização de dois termos que não apresentam consenso quanto a sua definição. Esse contexto foi determinante para a definição do desenho da pesquisa: i) período de análise e ii) seleção das variáveis.

As principais limitações no uso dessa ferramenta estão associadas à falta de indicadores relevantes, à perda de informações decorrentes da agregação de indicadores, inconsistências metodológicas nos métodos de ponderação e agregação (Bérenger \& Verdier-Chouchane, 2007:1261; Freudenberg, 2003:8).

Os procedimentos na construção dos índices agregados seguiram as fases de seleção, padronização, ponderação e agregação dos indicadores (Bérenger \& Verdier-Chouchane, 2007:1266; Blanc et al., 2008:254; Booysen, 2002:118). 


\subsection{Seleção dos Indicadores}

No âmbito educação é importante reconhecer a interação entre fatores geográficos, ecológicos, econômicos, sociais, culturais, políticos e institucionais, ou seja, o seu caráter multidimensional. Nota-se que todas essas dimensões são importantes para compreensão do conceito de educação, ao mesmo tempo em que se admite a dificuldade de selecionar indicadores capazes de representá-lo. Nem todos os aspectos da educação são passíveis de mensuração, o que torna qualquer tentativa de quantificá-lo, susceptível a críticas legítimas. Além disso, um mesmo conceito pode ser operacionalizado de diferentes formas.

Neste artigo buscou-se mensurar a educação em seus aspectos associados ao Programa Bolsa Família, mais especificamente aqueles implícitos na condicionalidade descrita no Art. $3^{\circ}$ da Lei $n^{\circ} 10.836$, de 9 de janeiro de 2004, o qual estabelece que a concessão de benefícios está vinculada entre outros quesitos, à frequência escolar de $85 \%$ (oitenta e cinco por cento) em estabelecimento de ensino regular. Assim, foram selecionadas as seguintes variáveis: Total de crianças e adolescentes de 6 a 17 anos no ensino público da educação, Quantidade de crianças e adolescentes de 6 a 17 anos acompanhados na frequência escolar, Número de Matrícula, Nota do IDEB $\left(5^{\circ}\right.$ e $9^{\circ}$ ano) e Taxa de aprovação.

A disponibilidade dos indicadores selecionados foi determinante para a definição do painel de dados adotado neste estudo, o qual cobriu o período 20052014, sendo composto por 27 Estados brasileiros.

Na tabela 1, a seguir, constam as estatísticas descritivas dos componentes do indicador educacional para o ano de 2014. Observa-se que em média a taxa de frequência para as crianças de 6 a 14 anos para os Estados foi de 92,68\% sendo maior que a taxa de frequência para os jovens de 15 a 17 anos que obteve somente $23,78 \%$. Logo a taxa de frequência dos jovens de 15 a 17 anos é inferior comparando com as crianças de 5 a 14 anos. 
Tabela 1 - Estatísticas Descritivas dos Indicadores Educacional Escolar no Brasilano 2014

\begin{tabular}{lccccc}
\hline \multicolumn{1}{c}{ Indicadores } & Média & Mínimo & Máximo & $\begin{array}{c}\text { Coeficiente } \\
\text { de variação }\end{array}$ & $\begin{array}{c}\text { Taxa de } \\
\text { Crescimento } \\
\mathbf{2 0 0 5 - 2 0 1 4}\end{array}$ \\
\hline $\begin{array}{l}\text { Taxa de frequência-6 a 14 } \\
\text { anos }\end{array}$ & 92,68 & 88,81 & 95,61 & $2 \%$ & $6,66 \%$ \\
$\begin{array}{l}\text { Taxa de frequência-15 a 17 } \\
\text { anos }\end{array}$ & 23,78 & 9,32 & 51,7 & $40 \%$ & $-21,85 \%$ \\
Nota do IDEB 5 ${ }^{\circ}$ ano & 5,19 & 4,25 & 6,25 & $12 \%$ & $45,03 \%$ \\
Nota do IDEB 9 ${ }^{\circ}$ ano & 4,13 & 3,3 & 4,85 & $11 \%$ & $24,3 \%$ \\
Taxa de Aprovação-Al & 91,93 & 86,2 & 97,9 & $4 \%$ & $15,43 \%$ \\
Taxa de Aprovação-AF & 83,3 & 69,3 & 95,3 & $7 \%$ & $10,7 \%$ \\
Taxa de Aprovação-EM & 78,65 & 66,6 & 86,9 & $6 \%$ & $9,03 \%$ \\
Matrícula- Al & 29147,63 & 21630 & 2009321 & $133 \%$ & $9,23 \%$ \\
Matrícula- AF & 1058204,15 & 92795 & 5537627 & $107 \%$ & $-14,8 \%$ \\
Matrícula- EM & 307458,52 & 22721 & 1928274 & $123 \%$ & $-8,08 \%$ \\
\hline
\end{tabular}

Fonte: Elaborado pelas autoras.

Nota-se que a taxa mínima para as crianças é de $88,81 \%$, sendo o Estado de Roraima que obteve a menor taxa e já a taxa dos jovens mínima é 9,32\%, sendo o Estado do Paraná que conseguiu essa taxa de frequência. Em relação ao máximo, 95,61\%, Rondônia que conseguiu a maior taxa de frequência para as crianças de 6 a 14 anos e o Acre com 51,7\% que obteve a maior taxa de frequência para os jovens de 15 a 17 anos.

Por outro lado, observa-se que a taxa de frequência de crianças de 6 a 14 anos obteve um aumento de 6,66\%. Já para os jovens de 15 a 17 anos ocorre uma redução de $21,85 \%$ na frequência escolar. São informações preocupantes, pois demonstram uma defasagem no sistema educacional. $O$ dado da queda de frequência escolar na faixa de 15 a 17 anos torna-se sério, uma vez que em uma sociedade cujos postos de trabalho requerem formação especializada, o mínimo que se exige é uma escolaridade de ensino médio completo. Para os jovens que não conseguirem formação adequada, será cada vez mais difícil atender as exigências do mercado de trabalho, consequentemente será mais difícil satisfazer suas necessidades pessoais e viver com dignidade.

Cardoso e Souza (2004) investigaram o impacto do Bolsa Escola sobre a frequência escolar e o trabalho infantil para meninos e meninas pertencentes a domicílios beneficiados ou não pelo Bolsa Escola para o ano de 2000. Os resultados indicam que o programa diminuía o tempo de trabalho da criança e aumentava a frequência escolar em cerca de três a cinco pontos percentuais.

Já em relação ao IDEB que é o principal indicador de qualidade de ensino básico no Brasil. $O$ indicador foi tomado como referência por ser um índice que combina desempenho nas provas nacionais (SAEB e Prova Brasil), com aprovação e passa a representar no âmbito do setor público importante mecanismo de monitoramento de qualidade dos sistemas escolares. Com uma escala que varia de 0 a 10, seria uma expressão de dois resultados desejáveis em um sistema de ensino - a aprovação escolar e o aprendizado em português e matemática. 
Verifica-se que os avanços na melhoria da qualidade da educação que resultam no IDEB, ocorrem em pequenos graus, o que se percebe pela diferença de 1,55 pontos em comparação com 2005 para o Estado de São Paulo que apresenta a maior nota entre os Estados para o ano de 2014, com 6,25 pontos. Já Amapá que é o Estado com a menor nota em 2014 com 4,25 pontos, apresenta uma diferença de 1,05 pontos em comparação a 2005. Este pequeno avanço evidencia que, embora estejam sendo feitas inúmeras mudanças educacionais no financiamento, planos de desenvolvimento e investimentos em todas as etapas da educação, os passos rumo aos índices de primeiro mundo são bem morosos.

De acordo com a lógica do Ministério da Educação é a de que para que o Brasil chegue à média 6,0 em 2021, período estipulado tendo como base o já citado bicentenário da Independência em 2022, cada sistema deve evoluir segundo pontos de partida distintos, e com esforço maior daqueles que partem em pior situação, com um objetivo implícito de redução da desigualdade educacional.

No que diz respeito à questão da qualidade, há razoável consenso, pelo menos entre pesquisadores da área, acerca da necessidade de se buscar um padrão mínimo de financiamento, um "custo aluno qualidade" que expressaria um conjunto de insumos necessários a um bom desenvolvimento do ensino (Vidal e Vieira, 2011). Com o IDEB, ampliam-se as possibilidades de mobilização da sociedade em favor da educação, uma vez que o índice é comparável nacionalmente e expressa em valores os resultados mais importantes da educação: aprendizagem e fluxo.

O terceiro componente é a taxa de aprovação que tem para anos iniciais (Al), anos finais (AF) e ensino médio (EM). Nota-se a média para os Estados foi maior nos anos inicias com $91,93 \%$ e sendo a menor média para ensino médio com $78,65 \%$. Para o Al o pior Estado foi Pará com 86,2\%, para AF foi Sergipe com 69,3\% e EM o Mato Grosso com 66,6\%. Para Al o melhor Estado foi Mato Grosso com 97,9\%, AF também Mato Grosso com 95,3\% e EM sendo Pernambuco com 86,9\%.

O quarto e último componente é o número de matrícula e observa-se que a média foi maior para os anos finais (AF) e menor para os anos iniciais (Al). Sendo Roraima que obteve o menor número de matrículas com valor para $\mathrm{Al}$ de 21630 e o maior foi São Paulo com 2009321.

Chama a atenção o fato de as matrículas para os anos finais e ensinos médios obtiveram uma redução de 14,8\% e 8,08\%, respectivamente. Já para anos iniciais um aumento de 9,23\%. Como consequência de experiências de repetência, baixo desempenho, atraso e discriminação, o abandono escolar cresce à medida que as séries avançam. Os indicadores de matrícula são importantes para dimensionar o tamanho do sistema educacional brasileiro.

Muitos estudos foram realizados com o objetivo de estimar as taxas de repetência e seus determinantes, foram feitos ou em níveis de agregação maiores, como um estado, ou utilizando bases de dados, como a Pesquisa Mensal de Emprego (PME), do Instituto Brasileiro de Geografia e Estatística (IBGE), nas quais não há variáveis relativas ao PBF (Klein e Ribeiro, 1991; Leon e Menezes-Filho, 2002; Duryea, 1998; Fletcher e Ribeiro, 1988). 
Oliveira e Soares (2013) demonstram que o PBF contribui para uma redução na probabilidade de repetência e observam que quanto maior a duração das aulas, menores as chances de repetência. Já turmas muito grandes apontam para maiores chances de reprovação. Como visto em Oliveira (2008) em termos de custo-benefício, uma política de ampliação da duração parece ser mais eficiente do que de redução da classe.

Uma política de universalização da conclusão da educação básica precisa criar mecanismos para diminuir as taxas de repetência e evasão e aumentar as de conclusão ao longo de um período de tempo. Nesse sentido, devem ser definidas metas que visam melhorar o sistema educacional e precisam apoiar-se nos diagnósticos já disponíveis para definir estratégias de intervenção e de monitoramento capazes de alterar os indicadores educacionais.

De acordo com o coeficiente de variação, é possível identificar em quais indicadores ocorre a maior heterogeneidade entre as unidades federativas brasileiras. Há uma elevada dispersão quanto ao número de matrícula, seja nos anos iniciais (133\%), matrícula do ensino médio (123\%) ou anos finais (107\%). Por outro lado, não há uma grande disparidade entre os estados quanto à taxa de frequência nos anos iniciais ( $2 \%$ ) e taxa de aprovação nos ensinos fundamental (Al e AF) e médio, com coeficientes $4 \%, 7 \%$ e $6 \%$, respectivamente.

\subsection{Padronização do indicador de educação (Scaling Method)}

Considerando as diferentes dimensões dos indicadores de educação optou-se pelo uso de padronização de modo a torná-los comparáveis com base em uma escala comum (Böhringer \& Jochem, 2006:3). Dados os diferentes métodos de padronização: standard deviation from the mean, distance from the group leader, distance from the mean, distance from the best and worst performers and categorical scale (Booysen, 2002,123-126; Freudenberg, 2003:10), optou-se pelo método standard deviation from the mean que expressa o quanto uma observação (dado) está acima ou abaixo da média em termos de unidades padronizadas de desvio e, assim, não é sensível a valores extremos (Blanc et al., 2008:254). Por este método, a transformação dos dados se dá pela expressão:

$$
z=\frac{X-\mu}{\sigma}
$$

Em que: $Z$ = escore padronizado, $X=$ escore bruto, $\mu=$ média populacional e $\sigma$ = desvio populacional.

\subsection{Ponderação (The weighting)}

Um pesquisador pode ou não atribuir pesos aos componentes de um índice agregado, dependendo da estrutura dos dados. Nardo, Saisana, Saltelli, \& Tarantola (2005:82) admitem duas situações em que equal weighting can be applied: quando todas as dimensões que irão compor o índice agregado apresentam o mesmo número de indicadores e quando existe alta correlação entre todos os indicadores, sem que haja redundância de informação. Além disso, o diagnóstico de colinearidade 
baseado no condition index ${ }^{2}$ sugere correlação entre todos os indicadores de educação (Tabela 2), o que nos levou a adotar a simple scheme based on equal weights, ou seja, descartar a ponderação.

Os valores médios de cada um dos índices são apresentados na Tabela 2. Considerando-se a escala de 0 a 1, os valores alcançados em ambos os índices são relativamente baixos. Nota-se que, em termos médios, os Estados analisados apresentaram níveis mais elevados de educação. No entanto, a heterogeneidade entre os Estados estudados também está aumentando, ou seja, educação está ocorrendo de forma desigual o que tende a acentuar as diferenças globais. É importante ressaltar que existem ameaças a educação que não foram contemplados na pesquisa devido a ausência de dados: caso dos elevados níveis de pobreza da população rural, baixo nível de capital humano, concentração de terras e vulnerabilidade às condições climáticas. Esses aspectos ressaltariam ainda mais a problemática observada na área de estudo.

Os indicadores discutidos representam aspectos distintos da educação escolar no Brasil. Com o propósito de tornar a análise mais objetiva optou-se pela agregação das informações em um índice. Assim, o Índice da Educação Escolar apresentado na Tabela 2, capta a situação de cada uma das 27 unidades federativas nas dimensões acesso à escola, participação estudantil, qualidade do ensino. Observa-se que a pior taxa de crescimento foi para o Estado de Alagoas com uma taxa de crescimento de $0,1 \%$ e o melhor Estado é Piauí que apresenta a maior taxa de crescimento com 20,6\%. O Estado que apresenta o melhor índice é São Paulo com valor 1 em 2014 apesar de ter apresentado uma taxa de crescimento só de 2,2\% e o pior Estado é Alagoas com 0,044 em 2006.

\section{Tabela 2- Índice de Educação por Unidade Federativa (nos anos 2006-2014- anos pares $^{3}$ )}

\begin{tabular}{ccccccc}
\hline ESTADOS & $\mathbf{2 0 0 6}$ & $\mathbf{2 0 0 8}$ & $\mathbf{2 0 1 0}$ & $\mathbf{2 0 1 2}$ & $\mathbf{2 0 1 4}$ & Taxa de Crescimento Média Anual \% \\
\hline Acre & 0,209 & 0,295 & 0,346 & 0,383 & 0,421 & 10,7 \\
Amapá & 0,221 & 0,225 & 0,265 & 0,286 & 0,333 & 4,8 \\
Amazonas & 0,171 & 0,237 & 0,298 & 0,387 & 0,448 & 16,6 \\
Pará & 0,115 & 0,149 & 0,303 & 0,333 & 0,351 & 13,7 \\
Rondônia & 0,240 & 0,271 & 0,299 & 0,394 & 0,457 & 7,8 \\
Roraima & 0,273 & 0,297 & 0,326 & 0,383 & 0,374 & 3,9 \\
Tocantins & 0,296 & 0,385 & 0,401 & 0,439 & 0,447 & 6,9 \\
Alagoas & 0,044 & 0,103 & 0,203 & 0,214 & 0,268 & 0,1 \\
Bahia & 0,174 & 0,210 & 0,328 & 0,384 & 0,425 & 13,0 \\
Ceará & 0,289 & 0,379 & 0,465 & 0,551 & 0,618 & 10,8 \\
Maranhão & 0,186 & 0,300 & 0,391 & 0,389 & 0,420 & 13,2 \\
Paraíba & 0,114 & 0,187 & 0,273 & 0,340 & 0,379 & 19,5
\end{tabular}

\footnotetext{
${ }^{2}$ A literatura estatística oferece vários métodos estatísticos para identificar a colinearidade entre variáveis. The condition index é um deles. Seu cálculo adota a expressão $\mathrm{Cl}=\sqrt{\lambda \mathrm{max} / \lambda \mathrm{i}}$, in which $\lambda$ max is the largest eigenvalue and $\lambda \mathrm{i}$ is the $\mathrm{i}^{\mathrm{th}}$ eigenvalue of the correlation matrix. $\mathrm{Cl}$ values between 10 and 100 indicate multi-collinearity. Dormann et al., (2013:32). Mais detalhes ver Belsley (1984).

3 Para fins de tornar a análise menos redundante e mais objetiva optamos por apresentar apenas anos pares, embora também tenha sido calculadas índices anuais para todo período 2005 a 2014.
} 
(conclusão)

\begin{tabular}{ccccccc}
\hline ESTADOS & $\mathbf{2 0 0 6}$ & $\mathbf{2 0 0 8}$ & $\mathbf{2 0 1 0}$ & $\mathbf{2 0 1 2}$ & $\mathbf{2 0 1 4}$ & Taxa de Crescimento Média Anual \% \\
\hline Pernambuco & 0,182 & 0,235 & 0,389 & 0,448 & 0,512 & 12,7 \\
Piauí & 0,119 & 0,183 & 0,311 & 0,350 & 0,406 & 20,6 \\
Rio Grande do Norte & 0,115 & 0,200 & 0,232 & 0,287 & 0,344 & 15,5 \\
Sergipe & 0,111 & 0,136 & 0,151 & 0,257 & 0,266 & 17,9 \\
Minas Gerais & 0,488 & 0,522 & 0,596 & 0,708 & 0,740 & 4,5 \\
Espírito Santo & 0,337 & 0,390 & 0,386 & 0,449 & 0,475 & 5,9 \\
Rio de Janeiro & 0,382 & 0,353 & 0,413 & 0,539 & 0,592 & 2,4 \\
São Paulo & 0,810 & 0,771 & 0,892 & 0,954 & 1,000 & 10,1 \\
Mato Grosso & 0,263 & 0,375 & 0,427 & 0,474 & 0,500 & 9,9 \\
Mato Grosso do Sul & 0,246 & 0,339 & 0,327 & 0,404 & 0,433 & 6,3 \\
Goiás & 0,365 & 0,382 & 0,416 & 0,564 & 0,617 & 6,0 \\
DF & 0,307 & 0,417 & 0,423 & 0,499 & 0,525 & 4,9 \\
Paraná & 0,450 & 0,522 & 0,501 & 0,586 & 0,630 & 5,0 \\
Rio Grande do Sul & 0,339 & 0,377 & 0,407 & 0,475 & 0,523 & 2,6 \\
Santa Catarina & 0,479 & 0,519 & 0,533 & 0,603 & 0,596 & \\
\hline
\end{tabular}

Fonte: Elaborado pelas autoras.

Logo, é necessário ter uma atenção maior para educação no Estado de Alagoas, pois se observa que em 2014 apresenta um índice de 0,268 e comparando com São Paulo que apresenta em 2006 o índice de 0,810. O mesmo ocorre para o Estado de Sergipe que não apresenta resultados bons para índice de educação, em 2014 apresenta um índice de 0,266. Em relação ao Ceará que apresenta um índice de 0,618 em 2014 e nota-se que foi melhor que muitos Estados e apresenta um taxa de crescimento de 10,8\%. O segundo melhor índice em 2014 é no Estado de Minas Gerais com 0,740 , mas com uma taxa de crescimento de apenas $4,5 \%$ para período. O Estado da Paraíba obteve uma alta taxa de crescimento de 19,5\%, apresentando em 2014 um índice de apenas 0,379. 
Gráfico 1 - Projeções 4

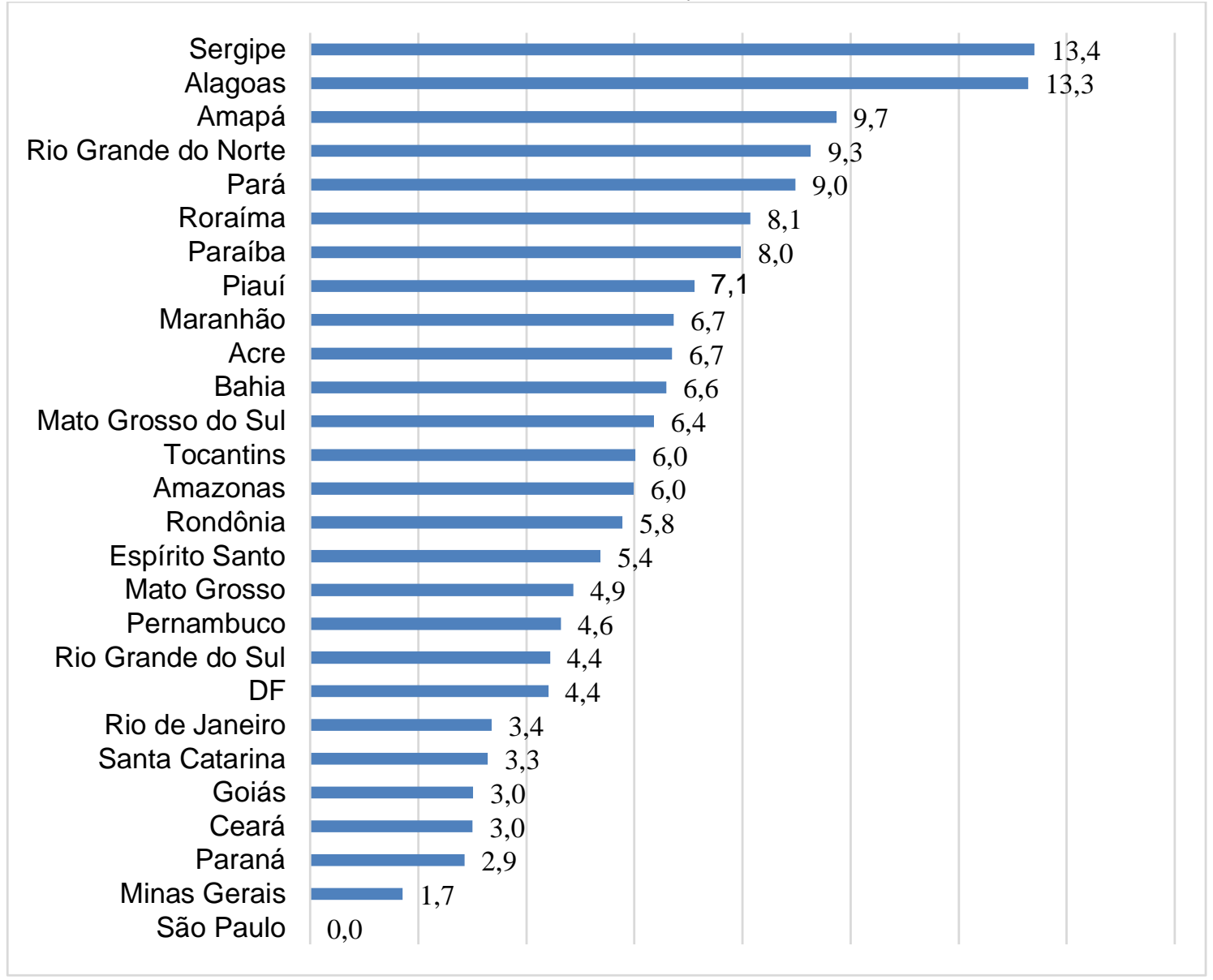

Fonte: Elaborado pelas autoras.

De um modo geral a situação da educação esta melhorando no Brasil como um todo. No entanto, em alguns Estados isso ocorre de forma lenta e os resultados verificados ainda não são bons o suficiente para que se alcance níveis mais elevados de educação. De fato no ritmo atual de mudanças serão necessários vários anos para se alcançar uma situação menos desigual entre as unidades federativas. Uma análise de projeções, gráfico 1, mostra que mesmo se os Estados crescessem a uma taxa igual ao Estado do Piauí, que apresenta a maior taxa de crescimento (20,6\%), ainda assim seriam necessários vários anos para chegar ao índice do estado brasileiro em melhor nível educacional em 2014 (Estado de São Paulo). É o caso de Sergipe e Alagoas, que precisariam de 13 anos e quatro meses e treze anos e três meses, respectivamente, para alcançar tal patamar.

\section{Relação entre o Índice de Educação e Programa Bolsa Família}

Com a estimação de modelos de regressão com dados em Painel espera-se verificar se os recursos do PBF sejam capazes de influenciar o comportamento dos indicadores educacionais nos Estados brasileiros.

Na tabela 3 estão apresentadas as estatísticas descritivas da variável do Programa Bolsa Família, número de famílias beneficiadas em 2014 e a taxa de

\footnotetext{
${ }^{4}$ Essa taxa corresponde ao período 2005 a 2014.
} 
crescimento entre 2005 e 2014. Observa-se que a maior taxa de crescimento é para Estado de Amapá com 385,84\% e ocorre uma redução de $9 \%$ para o Estado do Paraná. Piauí apresenta uma taxa de crescimento de 60,68\%. São Paulo com 53,63\%, Alagoas com $73,27 \%$ e Sergipe com $82,22 \%$. Já Santa Catarina obteve uma redução no número de famílias beneficiadas de $2,66 \%$.

Então, se o número de famílias possuírem uma relação com o índice de educação deve-se aumentar o número de pessoas beneficiadas do PBF nos piores Estados são Sergipe e Alagoas e ambos obtiveram um aumento no valor absoluto do número de famílias no período analisado de $82,22 \%$ e $73,27 \%$, respectivamente.

Tabela 3 - Estatísticas descritivas da variável do Programa Bolsa Família.

\begin{tabular}{|c|c|c|c|c|}
\hline \multirow[b]{2}{*}{ Estado } & \multicolumn{2}{|c|}{ Número de famílias (Mil) } & \multicolumn{2}{|c|}{$\begin{array}{l}\text { Valor acumulado no período 2005- } \\
\qquad 2014\end{array}$} \\
\hline & $\begin{array}{c}\text { Valor } \\
\text { Absoluto } \\
\text { (ano 2014) }\end{array}$ & $\begin{array}{c}\text { Taxa de } \\
\text { crescimento } \\
2005-2014\end{array}$ & $\begin{array}{l}\text { Valor absoluto } \\
\qquad(\text { Mil })\end{array}$ & Taxa de crescimento \\
\hline Acre & 78.561 & $101,91 \%$ & 610734 & $677,40 \%$ \\
\hline Alagoas & 439.655 & $73,27 \%$ & 3841942 & $774 \%$ \\
\hline Amapá & 55.527 & $385,84 \%$ & 417527 & $652 \%$ \\
\hline Amazonas & 358.516 & $143,45 \%$ & 2689480 & $650,17 \%$ \\
\hline Bahia & 1.808 .376 & $69,44 \%$ & 15656439 & $766 \%$ \\
\hline Ceará & 1.089 .813 & $46,79 \%$ & 9725126 & $792 \%$ \\
\hline DF & 86.524 & $59,76 \%$ & 826808 & $856 \%$ \\
\hline Espírito Santo & 190.049 & $18,16 \%$ & 1877373 & $888 \%$ \\
\hline Goiás & 336.606 & $80,13 \%$ & 2942948 & $774 \%$ \\
\hline Maranhão & 985.136 & $85,13 \%$ & 8242601 & $737 \%$ \\
\hline Mato Grosso & 186.272 & $56,98 \%$ & 1574674 & $745 \%$ \\
\hline Mato Grosso do Sul & 145.224 & $70,66 \%$ & 1244215 & $757 \%$ \\
\hline Minas Gerais & 1.143 .020 & $14,53 \%$ & 11130501 & $874 \%$ \\
\hline Pará & 887.426 & $162,79 \%$ & 6555574 & $639 \%$ \\
\hline Paraíba & 524.967 & $55,78 \%$ & 4492159 & $756 \%$ \\
\hline Paraná & 406.918 & $-9,00 \%$ & 4357376 & $971 \%$ \\
\hline Pernambuco & 1.150 .879 & $81,67 \%$ & 9928279 & $763 \%$ \\
\hline Piauí & 456.811 & $60,68 \%$ & 4022580 & $781 \%$ \\
\hline Rio de Janeiro & 827.847 & $173,46 \%$ & 6285669 & $659 \%$ \\
\hline $\begin{array}{l}\text { Rio Grande do } \\
\text { Norte }\end{array}$ & 362.805 & $50,65 \%$ & 3233548 & $791 \%$ \\
\hline Rio Grande do Sul & 434.715 & $9,19 \%$ & 4333632 & $897 \%$ \\
\hline Rondônia & $114 \cdot 170$ & $67,05 \%$ & 1046561,0 & $816,67 \%$ \\
\hline Roraima & 48.104 & $173,58 \%$ & 386.508 & $703,48 \%$ \\
\hline Santa Catarina & 137.970 & $-2,66 \%$ & 1399017 & $914 \%$ \\
\hline São Paulo & 1.327 .024 & $53,63 \%$ & 11508816 & $767 \%$ \\
\hline Sergipe & 281.231 & $82,22 \%$ & 2235210 & $695 \%$ \\
\hline Tocantins & 139.295 & $82,27 \%$ & 1195266 & $758 \%$ \\
\hline
\end{tabular}

Fonte: Elaborado pelas autoras. 
Com o objetivo de analisar a relação entre educação e programa bolsa família usa-se um painel data model. Em seguida quando as unidades de observação apresentam diferenças individuais (casos de Estados) e quando se quer captar variações que ocorrem ao longo do tempo (Marques, 2000:2) e controlar o viés de omissão de variáveis, time-invariant and individual-invariant factors.

Assume-se que dados em painel são mais adequados que modelos crosssection por meio do Teste Chow, observa-se que o valor da estatística F (tabela 4) e seu respectivo $p$-valore é menor que 0,05 , então rejeita a hipótese nula, isso significa que o modelo de Efeito fixo é melhor que o modelo pooled. Ao constatar que trata de um painel e não modelo pooled o próximo passo identificar se é efeito fixo ou efeito aleatório.

Foram testadas as três possibilidades de estimação de modelos de painel: modelo de regressão agrupada (pooled regression model), modelo de efeitos fixos (fixed effects models) e modelos de efeitos aleatórios (random effects models) e os resultados apontaram a escolha do modelo de efeitos fixos como a mais acertada. Foi feito o teste de Hausman e observa-se que o $p$ valore for menor que 0,05, logo o modelo de efeitos fixos é mais adequado que o modelo de efeitos aleatórios.

Outras razões reforçam essa especificação: características específicas de cada Estado que poderiam influenciar a Educação. Os testes de especificação do modelo indicaram, ainda, a inclusão de dummies de tempo. Utilizou-se assim, um time-fixed effect model.

Sendo:

$$
\log (\text { educacao })_{i t}=\beta \log (\operatorname{valorPBF})_{i t} 1+\alpha_{i}+\mu_{t}+\varepsilon_{i t}
$$

$\log (\text { educacao })_{\text {it }}=$ logaritmo do Indicador de educação, where $\mathrm{i}=$ state and $\mathrm{t}=$ year,

$\log (\text { valorPBF })_{\text {it }}=$ logaritmo do valor do Programa Bolsa Família.

$\beta=$ is the coefficient for that independent variable,

$\alpha_{i}$ - fixed effects, is the unknown intercept for each state (n country-specific intercepts). Fatores não observáveis que permanecem constantes ou aproximadamente constantes no curto prazo e variam entre Estados (captura a heterogeneidade entre Estados)

$\mu_{t}$ - time effects, is the unknown intercept for each year ( $t-1$ time periods intercepts). Fatores não observáveis que variam ao longo do tempo, mas são comuns a todos os Estados (captura a heterogeneidade ao longo do tempo).

cit - error term, idiosyncratic errors or idiosyncratic disturbances (these change across $t$ as well as across i)

The $i$ index shows the cross-sectional dimension (state), whereas the $t$ index shows the temporal dimension (year).

O uso de uma função logarítmica para representar o modelo traz vantagens como a interpretação dos coeficientes em termos de elasticidade, redução dos impactos negativos provocados por outliers e possibilidade de aumento na homocedasticidade dos erros (Khandker, 2005:278).

Considerando-se os pressupostos dos modelos de efeitos fixos (Wooldridge, 2010:251), se a estrutura dos dados sugere a sua adoção ao invés de pooled regression model or random effects model, existem razões que nos levam a acreditar que: existe 
uma heterogeneidade não observável ${ }^{5}$ entre os Estados que tende a permanecer constante no curto prazo e afeta a Educação;

Os resultados da estimação de diferentes modelos são apresentados na tabela 4. Todos os modelos foram estimados a partir de uma regressão, with autocorrelation and heteroskedasticity robust covariance matrix. De acordo com o modelo, o Programa Bolsa Família provoca impacto significativo no indicador educacional. Como se observa, um aumento de $1 \%$ no valor do PBF provoca, em média, um aumento de $0,52 \%$ no índice de educação. Portanto, o PBF proporciona ganohos educacionais.

Segundo a mesma linha, Romero e Hermeto (2009) avaliaram de forma pioneira os efeitos do PBF sobre os indicadores educacionais, quais sejam, a proporção de crianças no domicílio que deixaram de ir à escola no último mês; a razão de crianças no domicílio que evadiram do sistema de ensino entre 2004 e 2005; a proporção de crianças no domicílio que foram aprovadas entre 2004 e 2005; a razão de crianças que declararam somente estudar em relação às que trabalham e estudam, em comparação com aquelas que apenas trabalham e em relação àquelas que não trabalham e não estudam; e a proporção de crianças reprovadas entre 2004 e 2005. Esses indicadores foram desagregados segundo o sexo e recortes geográficos. Cabe observar que esses indicadores têm repercussão sobre a defasagem idade-série, embora os autores não investiguem o atraso escolar.

No período mais recente, Ribeiro e Cacciamali (2012) investigam os impactos do programa Bolsa-Família sobre a frequência escolar e a defasagem idade-série para o ano de 2006. Concluíram que ocorreu uma melhora nos indicadores, já que o programa exigia alto percentual de frequência às aulas, porém existem fatores correlacionados com a maior frequência às aulas, como a atitude da família em relação à escola, o capital cultural dessas famílias, que também devem contribuir para os bons resultados alcançados por essas crianças e adolescentes.

Tabela 4 - Resultado do Modelo Efeito Fixo*

\begin{tabular}{ccccccc}
\hline LnEducação & Coeficiente & Desvio Padrão & $\mathrm{t}$ & $\mathrm{p}>\mathrm{t}$ & Intervalo de confiança- 95\% \\
\hline InvalorPBF & 0.5201 & 0.059 & 8.81 & 0.000 & 0.3988 & 0.6414 \\
const & -11.2799 & 1.155 & -9.77 & 0.000 & -13.65414 & -8.9057 \\
Sigma_u & 0.65523 & & & & & \\
Sigma_e & 0.1969 & & & & & \\
rho & 0.9171 & & & & \\
Prob>F & 0.0000 & & & & \\
$\mathrm{~N}^{0}$ de observações & 269 & & & & \\
$\mathrm{~N}^{\circ}$ de grupos & 27 & & & & \\
\hline
\end{tabular}

*Heteroskedasticity-robust standard errors- $p<0.05$.

Fonte: Elaborado pelas autoras

Contudo, o impacto positivo do PBF se manifesta de forma distinta e tende a ser mais significativo, nas unidades federativas com índices educacionais mais baixos, como é possível observar no gráfico 2. O Estado de Alagoas obteve o maior impacto

\footnotetext{
${ }^{5}$ Assumimos como heterogeneidade não observável as variáveis não mensuráveis, mensuráveis com grande margem de erro ou não disponíveis. O modelo de efeitos fixos controla essa heterogeneidade e reduz o viés de omissão de variáveis relevantes na determinação do desenvolvimento rural (Yaffee, 2003:4)
} 
com valor de 1,19 e sendo o Estado com menor índice. Isso pode ser explicado pelo fato desses estados serem também os mais pobres e pelo maior potencial de políticas públicas como o PBF em locais onde há maior grau de pobreza. Nota-se que o impacto é menor no Estado de São Paulo, só o,16 e é o Estado que apresenta o melhor índice.

\section{Gráfico 2- Impacto do valor do PBF no Indicador de Educação}

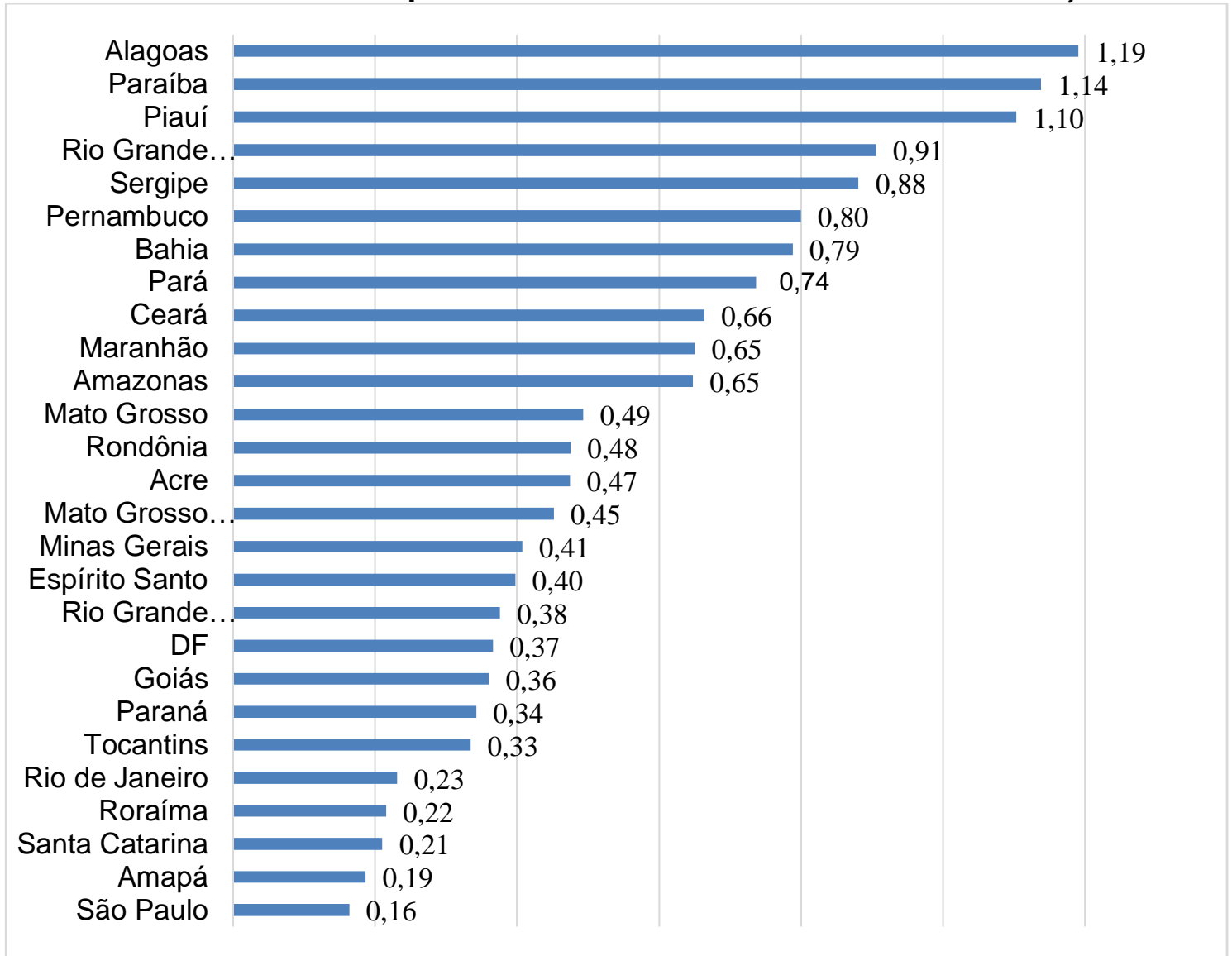

Fonte: Elaborado pelas autoras.

Mas se os gestores fizessem uma ponderação por Estado em relação a necessidade de mais investimento de acordo com indicador educacional devem aumentar o valor do PBF nos Estados que apresentam menor índice para diminuir a discrepância entre os Estados Brasileiros em relação a Educação.

\section{Considerações Finais}

Nessa conjuntura, a educação voltada para a construção do conhecimento e a formação da consciência. Para tanto, os dados sobre a realidade brasileira estão a exigir várias mudanças no âmbito cultural, social, econômico e político, sempre em busca de respostas e alternativas para que se atinja um nível de vida de melhor qualidade. O mais apontado instrumento de modificação é a educação.

Nesse sentido, surgiu como uma das alternativas de transformação o Programa Bolsa Família que tem como objetivo atrelar a transferência de renda à continuação da criança e adolescente na escola, ou seja, a contribuição do PBF na 
redução da desigualdade deve estar atrelada à melhoria dos indicadores de educação.

Os resultados deste artigo revelam que a situação da educação esta melhorando no Brasil como um todo. Mas, em alguns Estados isso ocorre de forma lenta e verifica-se são necessários vários anos para se alcançar uma situação menos desigual na educação entre as unidades federativas.

Cabe observar, no entanto, que o Programa Bolsa Família impacta significativamente na trajetória da Educação nos Estados Brasileiros. Porém, o impacto positivo do PBF se manifesta de forma distinta e tende a ser mais significativo, nas unidades federativas com índices educacionais mais baixos. Assim, o estabelecimento de um sistema escolar nacional e unificado tem um peso importante para redução da desigualdade educacional.

Em linhas gerais, mesmo reconhecendo o caráter exploratório do artigo, assume-se que no cenário descrito, no qual os Estados apresentam baixa e distinta qualidade de Educação, é questionável atribuir uma educação mais inclusiva e de melhor qualidade que melhorem o índice de Educação. Desperta os Estados para a necessidade de criar mecanismos que favoreçam a efetividade das políticas sociais realizadas pelo governo federal. Por fim, a complexidade da Educação requer a realização e integração de muitos outros estudos que identifiquem o alcance das mudanças que estão ocorrendo nas mais diferentes regiões do mundo sobre educação.

\section{REFERÊNCIAS}

BERHRMAN, J., SENGUPTA, P., \& TODD, P. Progressing through Progresa: An impact assessement of a school subsidy experiment in Mexico. PIER working paper 01-033, Penn Institute for Economic Research, Department of Economics, University of Pennsylvania. 2001.

BEHRMAN, J.R.; HODDINOTT, J. Programme Evaluation with Unobserved Heterogeneity and Selective Implementation: The Mexican PROGRESA Impact on Child Nutrition. Oxford Bulletin of Economics and Statistics, vol. 67, no. 4, 2005.

BÉRENGER, VALÉRIE, and AUDREY Verdier-Chouchane. "Multidimensional Measures of Well-Being: Standard of Living and Quality of Life Across Countries." World Development 35(7): 1259-76.

http://linkinghub.elsevier.com/retrieve/pii/S0305750X07000563 (September 14, 2014). 2007.

BLANC, Isabelle, DAMIEN Friot, MANUELE Margni, and OLIVIER Jolliet. "Weighting Approach." 260: 251-60. 2008.

BOOYSEN, Frederik. "AND EVALUATION OF COMPOSITE AN OVERVIEW INDICES." Social Indicators Research 59(2): 115-51. http://www.jstor.org/stable/27527024. 2002.

CACCIAMALI, M. C., TATEI, F., \& BATISTA, N. N. F. Pobreza, trabalho infantil e programa bolsa família. In: Cacciamali,M.C.\&José-Silva,M.F.,editors,A Construção 
da Igualdade de Gênero e Raça na América Latina no Século XXI: O Caso Brasil,pages 81-113. Suprema, São Carlos. 2009.

CARDOSO, E., and SOUZA, A., P. The Impact of Cash Transfers In Child Labor And School Attendance In Brazil. Working Paper No. 04-W07.Department Of Economics Vanderbilt University Nashville, Tn 37235.Www.Vanderbilt.Edu/Econ1. 2004.

DURYEA, S. Children's advancement through school in Brazil: the role of transitory shocks to household income. SSRN e Library, 1998.

FERRO, A. R. \& KASSOUF, A. L. Avaliação do impacto dos programas bolsa-escola sobre o trabalho infantil no Brasil. Pesquisa e Planejamento, 35:417-444.2005.

FLETCHER, P. R.; RIBEIRO, S. C. A educação na estatística nacional. In: SAWYER, D. O. (Org.). PNADs em foco: anos 80. Nova Friburgo: ABEP, 1988. p. 13-32. FREUDENBERG, Michael. Composite Indicators of Country Performance. Paris. http://dx.doi.org/10.1787/405566708255.2003.

FROTA, Maianara M. R.; ZIMMERMANN, Clóvis Roberto. O Brasil e as experiências internacionais de Programas de Transferência de Renda. In: XIII Congresso da Rede Mundial da Renda Básica, 2010, São Paulo. 13th International Conference of the Basic Income Earth Network. São Paulo : SínteseEventos, 2010. v. 1. p. 1-19.2010.

GLEWWE, P.; KASSOUF, A. O impacto do programa bolsa família no total de matrículas do ensino fundamental, taxas de abandono e aprovação, in 'Seminário Itaú Internacional de Avaliação Econômica de Projetos Sociais', São Paulo, 2010.

INSTITUTO BRASILEIRO DE GEOGRAFIA E ESTATÍ́STICA- IBGE. Censo Demográfico 2010.

JANVRY, Alain; FINAN, Frederic; SADOULET, Elisabeth. Evaluating Brazil's BolsaEscola Program: Impact on Schooling and Municipal Roles. Manuscrito. Berkeley,University of California at Berkeley.2006.

KHANDKER, S. R. “Microfinance and Poverty: Evidence Using Panel Data from Bangladesh." The World Bank Economic Review 19(2): 263-86. http://wber.oxfordjournals.org/cgi/doi/10.1093/wber/lhioo8 (August 20, 2014). 2005.

KLEIN, R.; RIBEIRO, S. C. O censo educacional e o modelo de fluxo: o problema da repetência. Revista brasileira de estatística, v. 1, n. 1, p. 5-45, 1991.

LEON, F. L. L.; MENEZES-FILHO, N. A. Reprovação, avanço e evasão escolar no Brasil. Pesquisa e planejamento econômico, v. 32, n. 3, 2002.

MARQUES, Luís David. “Modelos Dinâmicos Com Dados Em Painel: Revisão de Literatura." 2000. 
MELO, Raul da Mota Silveira; DUARTE, Gisléia Benini. Impacto do Programa Bolsa Família sobre a Frequência Escolar: o caso da agricultura familiar no Nordeste do Brasil. 2010.

MINISTÉRIO DO DESENVOLVIMENTO SOCIAL E COMBATE À FOME- MDS. Bolsa Família. 2016.

NEY, M. G. Educação e desigualdade de renda no meio rural brasileiro. Dissertação (Doutor em Economia Aplicada) - UNICAMP, Universidade de Campinas, Campinas, 124f. , 2006.

OLIVEIRA, J. M. Custo-efetividade de políticas de redução do tamanho da classe e ampliação da jornada escolar: uma aplicação de estimadores de matching. São Paulo: FEA/ USP, 31 $1^{\circ}$ Prêmio BNDES de Economia (20 lugar), 2008.

OLIVEIRA, L.; SOARES, S. O impacto do Programa Bolsa Família sobre a repetência: resultados a partir do cadastro único, projeto frequência e censo escolar. Brasília: Ipea, 2013 (Texto para Discussão, n. 1.814).

PONTILI. Rosangela. A infraestrutura escolar e as características familiares influenciando a frequência e o atraso no ensino fundamental. Piracicaba: ESALQ/USP, 2004.

PORTAL BRASIL-PB. Brasil está acima da média mundial na redução da mortalidade infantil, diz ONU. Objetivos do Desenvolvimento do Milênio. 2015.

PORTAL DO PLANALTO-PP. Tereza Campello faz um balanço do Plano Brasil sem Miséria no Bom Dia Ministro desta terça-feira (20/12). 2014.

RESENDE, A. C. C. Avaliando resultados de um programa de transferência de renda: O impacto do bolsa-família sobre os gastos das famílias brasileiras. Master's thesis, CEDEPLAR, Universidade Federal de Minas Gerais, Belo Horizonte.2006.

RIBEIRO, R. ; CACCIAMALI, M. C. Impactos do Programa Bolsa-Família Sobre os Indicadores Educacionais. Economia, Brasília(DF), v.13, n.2, p.415-446, mai/ago 2012.

ROMERO, J. A. R. \& HERMETO, A. M. Avaliação de impacto do programa bolsa família sobre indicadores educacionais: Uma abordagem de regressão descontínua. In ANPEC, editor, Anais do Encontro Nacional de Economia, Foz do Iguaçu. 2009.

SKOUFIAS, Emmanuel. "Progresa and its Impacts on the Welfare of Rural Households in Mexico". Research Report, no 139.Washington,DC, International Food Policy Research Institute.2005.

SOARES, J. F. Implementação de cotas na UFMG para alunos egressos de escolas públicas. In: Universidade e democracia:experiências e alternativas para a ampliação do acesso à Universidade pública. Belo Horizonte: Editora UFMG, 2004. 
SOARES, F. V.; RIBAS, R. P. \& OSÓRIO, R. G. “Avaliando o impacto do Programa Bolsa Família: uma comparação com programas de transferência condicionada de renda de outros países". Centro Internacional da Pobreza, IPEA/PNUD. 2010.

VIDAL, E. M. VIEIRA, S. L. Gestão educacional e resultados no Ideb: um estudo de caso em dez municípios cearenses. Est. Aval. Educ., São Paulo, v. 22, n. 50, p. 419434, set./dez. 2011.

WOOLDRIDGE, Jeffrey M. Econometric Analysis of Cross Section and Panel Data. MIT Press. 2010.

Patrícia Verônica Pinheiro Sales Lima. Doutora em Economia e Professora do Programa de Pós-graduação em Desenvolvimento e Meio Ambiente (PRODEMA) da Universidade Federal do Ceará - UFC. patricialima@ufc.br

Joyciane Coelho Vasconcelos. Doutoranda em Desenvolvimento e Meio Ambiente (UFC). Mestre em Economia Rural/UFC. Especialista em Adminstração Financeira. Graduada em Ciências Econômicas pela Universidade Federal do Ceará- Campus Avançado de Sobral. Endereço: Universidade Federal do Ceará, Centro de Ciências Agrárias, Departamento de Economia Agrícola. AC Campus do Pici. CEP: 60440970 - Fortaleza, CE - Brasil. joyciane.c.v@gmail.com

Como citar: LIMA, Patrícia Verônica Pinheiro Sales; VASCONCELOS, Joyciane Coelho. Programa Bolsa Família e educação escolar: uma abordagem de dados em painel. Redes, Santa Cruz do Sul, v. 24, n. 1 , p. 335-355, jan. 2019. ISSN 1982-6745. Disponível em: https://doi.org/10.17058/redes.v24i1.11692. 